\title{
Solid Pseudopapillary Neoplasm of the Pancreas: Clinicopathologic Feature, Risk Factors of Malignancy, and Survival Analysis of 53 Cases from a Single Center
}

\author{
He Song, Ming Dong, Jianping Zhou, Weiwei Sheng, Banghua Zhong, and Wei Gao \\ Department of Gastrointestinal Surgery, The First Hospital of China Medical University, Shenyang 110001, China \\ Correspondence should be addressed to Ming Dong; dongming@cmu.edu.cn
}

Received 14 May 2017; Revised 9 July 2017; Accepted 31 July 2017; Published 28 September 2017

Academic Editor: Kosei Maemura

Copyright (C) 2017 He Song et al. This is an open access article distributed under the Creative Commons Attribution License, which permits unrestricted use, distribution, and reproduction in any medium, provided the original work is properly cited.

Introduction. Solid pseudopapillary neoplasm (SPN) of the pancreas is a rare tumor of low malignant potential. The aim of this study was designed to evaluate the clinicopathologic feature, predictive factors of malignancy, and survival from experience of a single center. Methods. 53 consecutive patients who underwent surgery for a pathologically definitive SPN were retrospectively reviewed. Results. A total of 53 cases included 7 male cases and 46 female cases with the median age of 35.4 years (14-67). Abdominal pain and mass were the most common clinical presentations. The radiological presentations were consistent with solid and cystic pattern in 18 cases, solid pattern in 25 cases, and cystic pattern in 10 cases. The predominant location of tumor was pancreatic body and tail. The mean size of the tumors was $6.4 \mathrm{~cm}$. Aggressive en bloc resection combined with organ-preserving should be indicated whenever feasible. Follow-up information was available for 48 patients with a median follow-up time of 48 months. The 5-year disease-specific survival was $95.7 \%$. Incomplete capsule was not only the predictive factor of malignancy but also the significant predictor of disease-specific survival. Conclusion. Incomplete capsule may suggest a malignant SPN and a prognostic indicator of disease-specific survival. We recommend that surgeons consider a more radical resection with an incomplete capsule of tumor.

\section{Introduction}

Solid pseudopapillary neoplasm (SPN) is a rare pancreatic tumor predominantly affecting young women with low malignant potential [1] and was first reported by Frantz in 1959 [2]. It usually has a favorable prognosis, with just over $95 \%$ of patients reported as being disease-free after surgical resection and with less than $2 \%$ mortality [3]. The aim of this study was designed to evaluate the clinicopathologic feature, predictive factors of malignancy, and survival from 13-year experience of a single center.

\section{Materials and Methods}

Between January 2004 to January 2017, 53 consecutive patients with a definitive pathological diagnosis of SPN at the Department of General Surgery, The First Hospital of China Medical University, were retrospectively reviewed. Patients' demographic, clinical presentation, radiological details, surgical data, pathological features, long-term survival, and other relevant data were extracted from hospital records and evaluated. Outpatient records combined with telephone interviews were used for follow-up. The criteria of SPN were defined as malignant if it demonstrated extrapancreatic invasion, distant metastases, pancreatic parenchymal invasion, peripancreatic fat tissue infiltration, lymph node involvement, capsular invasion, or perineural or vascular invasion [4-7]. Patient status was due to the time of last follow-up as follows: no evidence of disease, alive with disease, dead of disease, surgical mortality, and dead of other causes. Survival time was defined as starting from the date of first operation until end of follow-up due to either death or end of data collection. Only "dead of disease" was considered an event in the analysis of disease-specific survival. Descriptive statistics were represented as mean \pm standard deviation [SD] or as the median (range) or as 
proportion. The characteristics of the 2 patient groups (with benign and malignant SPNs) were compared. Univariable analysis was performed using $\chi^{2}$ test for categorical variables and Student's $t$-test for continuous variables. The KaplanMeier method was used to estimate survival. The log-rank test was used to analyze differences in patient survival. $P$ value of less than 0.05 was considered significant. All statistical analyses were performed with the Statistical Package for Social Sciences (SPSS) 16.0 for Windows (Chicago, Illinois).

\section{Results}

3.1. Common Information. A total of 53 cases included 7 male cases and 46 female cases and the ratio of male/female was $1: 6.6$. Meanwhile the youngest is 14 -year-old and the eldest is 67-year-old and the average age was $35.4 \pm 13.4$ years. The average of BMI was $23.7 \pm 2.4$ (18.6 to 28.5). There were 8 patients infected with Hepatitis B virus (HBV) and 2 patients infected with Hepatitis $\mathrm{C}$ virus (HCV).

3.2. Clinical Manifestations. The clinical presentation was upper abdominal pain in 20 cases (37.7\%), abdominal distention in 9 cases $(20.0 \%)$, abdominal mass in 16 cases $(30.2 \%)$, incidental detection in 21 cases (39.6\%), nausea and vomiting in 6 cases (11.3\%), back pain in 3 cases $(5.7 \%)$, and hematuria in 1 case $(1.9 \%)$. No one presented the obstructive jaundice. The symptoms were nonspecific, and coexistence of two or more symptoms was often found.

3.3. Preoperative Examination. The level of tumor markers, including AFP, CEA, CA199, CA125, and CA242, was slightly increased in 6 patients, but none were diagnosed as malignant SPN. In addition, according to our hospital cases, the most preoperative laboratory tests were within normal limits.

The three most common forms of abdominal imaging were CT, ultrasound, and MRI, and accounted for 50 patients (94.3\%), 48 patients (90.6\%), and 10 patients (18.9\%), respectively. The radiological appearance of SPN is typically characterized by well-encapsulated, heterogenous (solid and cystic) mass, whose solid component was enhanced on the arterial and venous phase but lower than normal pancreatic tissue. The radiological presentations were consistent with solid and cystic pattern in 18 cases, solid pattern in 25 cases, and cystic pattern in 10 cases. Calcification in the tumor and dilated main pancreatic duct were reported for 15 patients and 3 patients. There were 45 cases completely encapsulated and 8 cases incompletely encapsulated in the radiological image. 7 cases of incomplete capsule were malignant. Preoperative percutaneous biopsies and endoscopic ultrasound-fine needle aspiration (EUS-FNA) were performed in 2 cases and 3 cases. Among these cases, 3 cases were diagnosed as SPN, 1 case was diagnosed as dubious pancreatic tumor tissue, and another 1 case was misdiagnosed as pancreatic islet cell tumor.

15 cases $(28.3 \%)$ were distributed on the pancreatic head, 32 cases $(60.4 \%)$ were distributed on the pancreatic body and tail, 5 cases $(9.4 \%)$ were distributed on the pancreatic neck, and 1 case $(1.8 \%)$ was on the extrapancreatic site (located at site surrounded by the left kidney, spleen, and pancreatic tail). The mean size of the tumors was $6.4 \pm 3.5 \mathrm{~cm}(2-14 \mathrm{~cm})$.
3.4. Surgery. All the 52 cases had $R_{0}$ resection. 6 cases underwent pancreaticoduodenectomy, 3 cases underwent pyloruspreserving pancreaticoduodenectomy, 3 cases underwent duodenum-preserving pancreatic head resection, 2 cases underwent cases of middle pancreatectomy, 15 cases underwent distal pancreatectomy with preserving-spleen, 13 cases underwent distal pancreatectomy with splenectomy, and 10 cases underwent enucleation. In 1 case of gastric involvement, another case of renal involvement, and 2 cases of transverse colon involvement, we performed tumor resection plus subtotal gastrectomy, nephrectomy, and colectomy. One case had palliative operation $\left(R_{2}\right)$. Radical resection was impossible because massive superior mesenteric vein involvement made mass reduction and graft reconstruction unfeasible. Total surgical period was $3.9 \pm 1.2$ hours ( $1-8.5$ hours). Blood transfusion was given to 3 patients in the volume of 2 units, 2 units, and 4 units, respectively. No postsurgical adjuvant therapy was administered to any patient.

There were no surgical mortalities. Postsurgical complications occurred in 14 patients. The most common complication was pancreatic fistula (9 cases), followed by intraabdominal abscess ( 4 cases) and gastric fistula (1 case). These complications were all resolved by conservative therapy. Statistics all above was listed in Table 1.

3.5. Pathological and Immunohistochemical Characteristics. The typical gross appearance of SPN is well capsulated and demarcated from the pancreas, with a mixture of solid, cystic component in various proportions.

Microscopically, tumor cells arranged around fibrovascular stalk forming pseudopapillary pattern, focal areas of hemorrhage, and necrosis could usually be found. Immunohistochemical staining showed that alpha 1-antichymotrypsin (AACT), Vinmentin, alpha 1-Antitrypsin (AAT), NeuronSpecific Enolase (NSE), Progesterone Receptor (PR), Synaptophysin, and so forth appear to have positive expression mostly and the positive rates for them were $95.7 \%(45 / 47)$, $88.1 \%$ (37/42), 82.5\% (33/40), 70\% (28/40), 63.9\% (23/36), and $55.3 \%(21 / 38)$, respectively, as is shown in Table 2. Immunohistochemical staining of Ki-67 was detected in 8 patients, 5 cases were expressed positive, and 4 cases of the noted 5 patients were confirmed to be malignant; Ki-67 immunoreactivity of 4 malignant cases was less than $1 \%$.

3.6. Characteristics of Malignant SPN. 53 cases were pathologically confirmed as SPN, 10 cases (18.9\%) were diagnosed as malignant, as follows: vascular infiltration was identified in 1 patient, pancreatic parenchymal invasion concurrent with peripancreatic fat tissue infiltration was in 4 cases, adjacent organ invasion was in 4 cases, and perineural invasion was in 1 case. One case of pancreatic parenchyma invasion suffered from recurrence and underwent a second operation after 8 years of the first resection. None had lymph node metastasis. No tumors presented severe nuclear atypia or a high mitotic rate. Table 3 compared and summarized the characteristics between patients with benign and malignant tumor.

3.7. Predictive Factors of Malignancy. There is no statistical difference in the age, sex, symptom, serum tumor marker, 
TABLE 1: Clinical characteristics of SPN patients.

\begin{tabular}{|c|c|c|}
\hline Parameter & Patient number $(n=53)$ & $\%$ \\
\hline Age (year, average) & 35.4 & \\
\hline \multicolumn{3}{|l|}{ Gender } \\
\hline Female & 46 & $86.8 \%$ \\
\hline Male & 8 & $13.2 \%$ \\
\hline BMI (average) & 23.7 & \\
\hline \multicolumn{3}{|l|}{ Hepatitis type } \\
\hline Hepatitis B virus & 8 & $15.1 \%$ \\
\hline Hepatitis $C$ virus & 2 & $3.8 \%$ \\
\hline \multicolumn{3}{|l|}{ Clinical presentation } \\
\hline Abdominal pain & 20 & $37.7 \%$ \\
\hline Abdominal distention & 9 & $20.0 \%$ \\
\hline Abdominal mass & 16 & $30.2 \%$ \\
\hline Incidental detection & 21 & $39.6 \%$ \\
\hline Nausea and vomiting & 6 & $11.3 \%$ \\
\hline Back pain & 3 & $5.7 \%$ \\
\hline Hematuria & 1 & $1.9 \%$ \\
\hline \multicolumn{3}{|l|}{ Component of tumor } \\
\hline Solid and cystic & 18 & $34.0 \%$ \\
\hline Solid & 25 & $47.2 \%$ \\
\hline Cystic & 10 & $18.9 \%$ \\
\hline Calcification & 15 & $28.3 \%$ \\
\hline Dilated main pancreatic duct & 3 & $5.7 \%$ \\
\hline \multicolumn{3}{|l|}{ Tumor location } \\
\hline Head & 15 & $28.3 \%$ \\
\hline Body & 32 & $60.4 \%$ \\
\hline Neck & 5 & $9.4 \%$ \\
\hline Extrapancreatic site & 1 & $1.8 \%$ \\
\hline Tumor size $(\mathrm{cm})$ & 6.4 & \\
\hline \multicolumn{3}{|l|}{ Operation type } \\
\hline Pancreaticoduodenectomy & 6 & $11.3 \%$ \\
\hline Pylorus-preserving pancreaticoduodenectomy & 3 & $5.7 \%$ \\
\hline Duodenum-preserving pancreatic head resection & 3 & $5.7 \%$ \\
\hline Middle pancreatectomy & 2 & $3.8 \%$ \\
\hline Distal pancreatectomy with preserving-spleen & 15 & $28.3 \%$ \\
\hline Distal pancreatectomy with splenectomy & 13 & $24.5 \%$ \\
\hline Enucleation & 10 & $18.9 \%$ \\
\hline Palliative operation & 1 & $1.9 \%$ \\
\hline Surgical period (hour average) & 3.9 & \\
\hline \multicolumn{3}{|l|}{ Postsurgical complications } \\
\hline Pancreatic fistula & 9 & $17.0 \%$ \\
\hline Intra-abdominal abscess & 4 & $7.5 \%$ \\
\hline Gastric fistula & 1 & $1.9 \%$ \\
\hline \multicolumn{3}{|l|}{ Capsule pattern } \\
\hline Complete capsule & 45 & $84.9 \%$ \\
\hline Incomplete capsule & 8 & $15.1 \%$ \\
\hline Benign tumor & 43 & $81.1 \%$ \\
\hline Malignant tumor & 10 & $18.9 \%$ \\
\hline Vascular infiltration & 1 & \\
\hline Pancreatic parenchymal invasion & 4 & \\
\hline Adjacent organ invasion & 4 & \\
\hline Perineural invasion & 1 & \\
\hline
\end{tabular}


TABLE 2: Immunohistochemical staining.

\begin{tabular}{lccc}
\hline Parameter & Positive & Total & $\%$ \\
\hline AACT & 45 & 47 & $95.7 \%$ \\
Vinmentin & 37 & 42 & $88.1 \%$ \\
AAT & 33 & 40 & $82.5 \%$ \\
NSE & 28 & 40 & $70 \%$ \\
PR & 23 & 36 & $63.9 \%$ \\
Synaptophysin & 21 & 38 & $55.3 \%$ \\
Ki-67 & 5 & 8 & $62.5 \%$ \\
\hline
\end{tabular}

TABLE 3: Predictive factors of malignant SPN.

\begin{tabular}{|c|c|c|c|}
\hline Factor & Benign & Malignant & $P$ value \\
\hline Average age & $34.1 \pm 12.0$ & $41.0 \pm 17.8$ & NS \\
\hline Average BMI & $23.6 \pm 2.5$ & $24.1 \pm 1.9$ & NS \\
\hline Gender & & & NS \\
\hline Male & 6 & 1 & \\
\hline Female & 37 & 9 & \\
\hline Symptoms & & & NS \\
\hline Present & 27 & 4 & \\
\hline Absent & 16 & 6 & \\
\hline Serum tumor marker & & & NS \\
\hline Elevated & 37 & 10 & \\
\hline Normal & 6 & 0 & \\
\hline Average tumor size $(\mathrm{cm})$ & & & NS \\
\hline$<5 \mathrm{~cm}$ & 20 & 2 & \\
\hline$>5 \mathrm{~cm}$ & 23 & 8 & \\
\hline Tumor location & & & NS \\
\hline Head & 12 & 3 & \\
\hline Body and tail & 27 & 5 & \\
\hline Neck & 4 & 1 & \\
\hline Extrapancreatic site & 0 & 1 & \\
\hline Calcification condition & & & NS \\
\hline Calcification & 11 & 4 & \\
\hline Noncalcification & 32 & 6 & \\
\hline Component of tumor & & & NS \\
\hline Solid and cystic & 13 & 5 & \\
\hline Solid & 19 & 4 & \\
\hline Cystic & 11 & 1 & \\
\hline Pattern of capsule & & & $P<0.001$ \\
\hline Complete capsule & 42 & 3 & \\
\hline Incomplete capsule & 1 & 7 & \\
\hline
\end{tabular}

tumor size, tumor location, calcification, and component of tumor. However, we found that incomplete capsule is significantly more in the malignant group $(P<0.001)$. The typical CT image in our cases series including complete and incomplete capsule was in Figures 1(a) and 1(b).

3.8. Follow-Up Results. Follow-up data were collected by telephone or outpatient interview. Follow-up information was available for 48 patients with a median follow-up time of 48 months (3-123 months). 45 patients were alive at last follow-up, and 43 patients were without evidence of disease, including 3 patients after resection of adjacent organs. Two patients were alive with disease. One patient suffered from recurrence, and this patient who underwent enucleation developed tumor recurrence after 8 years and had a second operation. The other one who had the palliative operation survived well for 65 months. The disease-free survival was 91.7\%. Three patients died: 2 died of SPN and 1 died of a traffic accident. Five patients were lost to follow-up. The 3-, 5-, and 10-year disease-specific survival were 100\%, 95.7\%, and $95.7 \%$. By univariate analyses, incomplete capsule $(P=$ 0.019 ) was significant predictor of disease-specific survival 


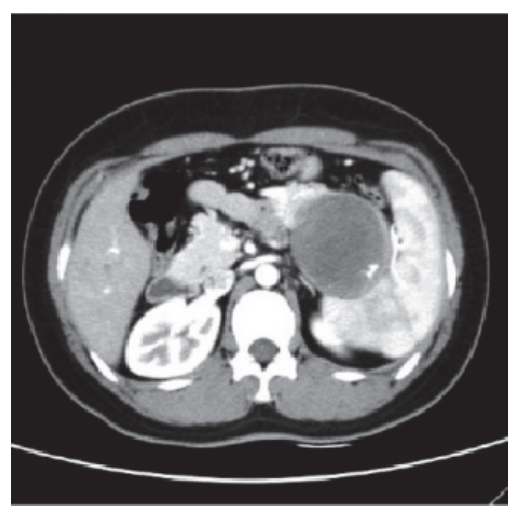

(a)

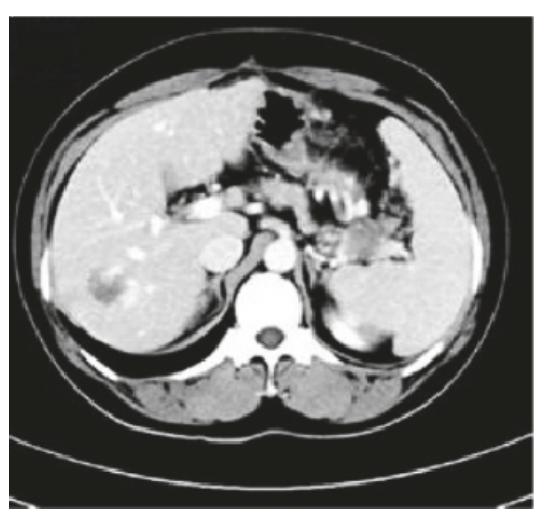

(b)

Figure 1: (a) The SPN locating at the tail of pancreas with complete capsule was confirmed to be benign. (b) The SPN locating at the tail of pancreas with incomplete capsule was confirmed to be malignant in the infiltrative growth pattern. Splenic vessels were invaded by the tumor. The mass at the right lobe of liver was confirmed to be hepatic hemangioma.

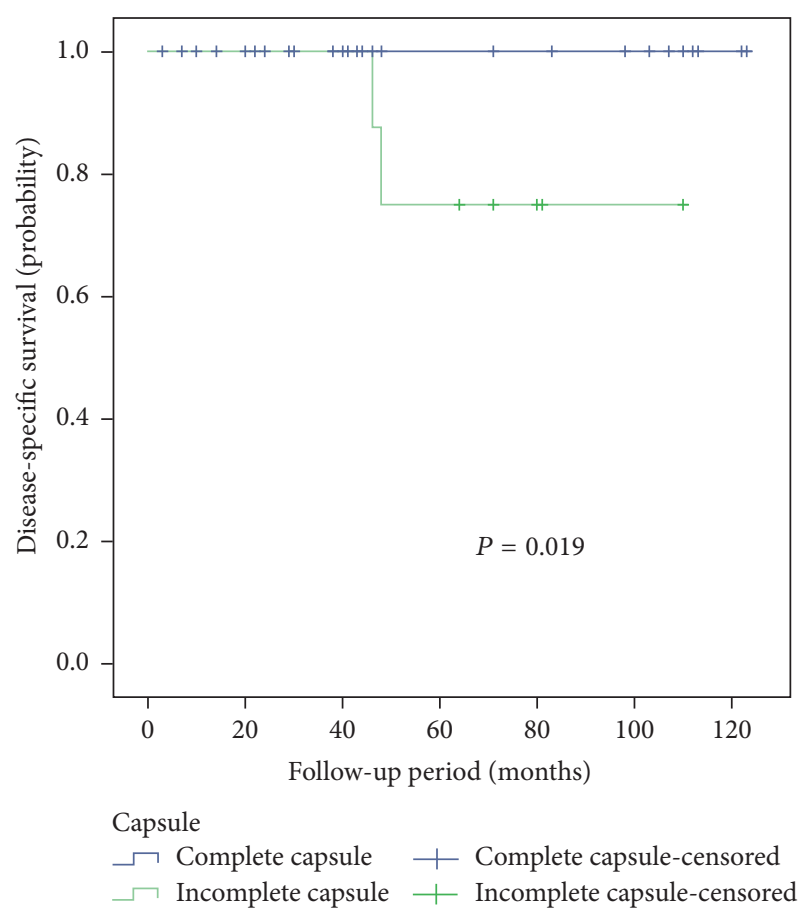

FIGURE 2: Cumulative Kaplan-Meier survival curve associated with capsule in SPN patients.

(Figure 2), but not age, sex, symptom, serum tumor marker, tumor size, tumor location, calcification, and component of tumor.

\section{Discussion}

SPN frequently occurs in young women. Law et al. [3] had performed a systematic review for English literature that indicated that female cases of SPN occupied $87.8 \%$ in the total of 2408 patients; the average age was $28.5 \pm 13.7$ years. In our study, female comprised $86.8 \%$ of total of 53 cases; meanwhile the average age of 53 cases was $35.4 \pm 13.4$ years. Although female patients with SPN outnumbered male patients, there were no gender-specific trends in expression of sex hormone receptor protein or clinicopathologic characteristics $[8,9]$. Machado et al. [10] analyzed 34 cases including 7 male patients retrospectively and found that male patients with more aggressive behavior had distinct patterns of onset and aggressiveness compared to female patients. However, in our study, there was no correlation between malignancy and the age, sex, or BMI. The average of BMI of 53 cases was $23.7 \pm 2.4$ (18.6 to 28.5 ), so there was no connection between obesity and SPN. There were 8 patients infected with Hepatitis $\mathrm{B}$ virus (HBV) and 2 patients infected with Hepatitis C virus (HCV). Some study [11] reported that $62.5 \%$ of SPN patients were infected with $\mathrm{HBV}$, which may correlate with the pathogenesis of SPN, but our study could not demonstrate this association.

The presentation of SPN is usually nonspecific. Similar to some study $[1,3]$, our data showed that abdominal pain was found in $37.7 \%$ of the patients and abdominal mass was seen in $30.2 \%$ of the patients. Many patients (39.6\%) had no symptoms, and tumor was incidentally detected by imaging or medical examination. For most tumor size is big with the average diameter of $6.4 \pm 3.5 \mathrm{~cm}$; some patients presented symptoms of tumor compression affecting the alimentary tract, such as nausea and vomiting. However, there was no correlation between symptom and tumor size $(P>0.05)$. It is necessary to note that even if the tumor was located at the head of pancreas, no case caused obstructive jaundice due to the tumor exophytic growth way, which was consistent with some study $[1,3]$.

In the radiological image, SPN typically represented wide-ranging appearance from solid to cystic, a well capsulated mass, with solid and cystic component. In our study, concerning the solid component, the average of $\mathrm{Hu}$ value of plain CT scan, arterial phase, and venous phase was $37.8 \mathrm{Hu}$, $58.1 \mathrm{Hu}$, and $63.4 \mathrm{Hu}$. The $\mathrm{Hu}$ value between plain phase and arterial phase/venous phase was statistically significant $(P<0.05)$; however, the $\mathrm{Hu}$ value between arterial phase 
and venous phase was not $(P>0.05)$. Calcification may be present in some cases, whereas dilation of pancreatic duct is rare. MR imaging displaying intratumoral hemorrhage and the capsule of the SPN is better than CT [12]. But MRI data in our study was scarce. There was no correlation between malignancy and component of tumor and calcification in our study, consistent with [13]; however Hwang et al. regarded proportion of solid component was associated with malignancy [6]. Preoperative percutaneous biopsies and endoscopic ultrasound-fine needle aspiration (EUS-FNA) may establish an accurate preoperative diagnosis. Some studies $[14,15]$ had confirmed that EUS-FNA was a reliable tool that significantly increased diagnostic accuracy by characterizing the cytomorphological features. However, the procedure may cause tumor cell dissemination [16]. The 5 cases in our series did not experience any complications. There were 10 cases diagnosed malignant, and 7 of them were with incomplete capsule. Incomplete capsule defined a capsule that did not surround the entire periphery of the tumor in the radiological morphology. In the 7 tumors of incomplete capsule, there were 2 cases of exophytic growth pattern and 5 cases of infiltrative growth pattern. Most benign cases or cases with complete capsule exhibited exophytic pattern. Infiltrative growth pattern might cause the disruption of capsule and indicate the malignant behavior. Capsule morphology was significant between two groups $(P<0.05)$, consistent with some studies $[17,18]$. Notably, two patients who died of SPN had the incomplete capsule with infiltrative growth pattern.

In laboratory tests, parameters were commonly within normal scope, so the routing laboratory parameters and tumor markers are of no help.

In our study, the predominant localization of tumor is the body and tail of the pancreas, followed by the head and the neck. It is worth mentioning that there was one case in the extrapancreatic sites, located at site surrounded by the left kidney, spleen, and pancreatic tail. This malignant tumor invaded the left kidney, caused the symptom of back pain and hematuria, and finally was resected with both tumor and kidney. The most common extrapancreatic sites were the mesocolon and ovary [19], sometimes even in the testicle [20]. It is cautious to explore carefully for ectopic pancreatic neoplasm to avoid misdiagnosis.

The preferred treatment of SPN is the surgery. Operational style depended on tumor's size, tumor's location, and intraoperative frozen section examination. Due to the low malignancy of tumor, organ-preserving operation should be performed whenever feasible [21]. For 3 cases of pyloruspreserving pancreaticoduodenectomy, it was found that tumor invaded pancreatic parenchyma or duodenum in the intraoperative frozen section examination, so pyloruspreserving pancreaticoduodenectomy was indicated. For 3 cases of duodenum-preserving pancreatic head resection, tumors were located at the head of pancreatic parenchyma, and it was unfeasible for enucleation because of damage of main pancreatic duct or superior mesenteric vessel. For the tumor on the pancreatic neck, 2 cases underwent central pancreatectomy. For the tumor on the pancreatic body and tail, distal pancreatic resection was carried out with splenectomy in 13 cases and with preserving-spleen in 15 cases. For the tumor's ectogenous growth tumor and complete amicula, away from the pancreatic duct and important pancreatic blood vessels, enucleation was carried out in 10 cases. One case had palliative operation $\left(R_{2}\right)$ because of massive superior mesenteric vein involvement. However, invasion to the portal vein or superior mesenteric artery should not be a contradiction of surgery [1]; some study carried out the vascular reconstruction with vein grafts after en bloc resection and had a favorable survival. For the patients whose tumor could not be completely resected, palliative surgery also provided a good survival. In addition, one case of SPN was found outside the pancreas, so careful exploration is particularly important in the operation.

Recurrence rate is estimated in $10-15 \%$ of patients after resection [22], whereas only 1 patient (1.9\%) suffered from tumor recurrence in our study. The patient underwent the second enucleation during 96-month disease-free period during follow-up. Our series included 1 case of gastric involvement, another case of renal involvement, and 2 cases of transverse colon involvement. We performed tumor resection plus subtotal gastrectomy, nephrectomy, and colectomy resulting in good survival. Aggressive en bloc resection should be always be attempted including resection of concomitant metastases and infiltrative organ.

SPN rarely had lymph node involvement, no cases had lymph node involvement in our series, and extensive lymphatic dissection is not necessary.

Diagnosis of SPN mainly depends on the pathology and immunohistochemistry. In addition, our study showed the positive expression rates of Vinmentin, AACT, AAT, NSE, Synaptophysin, and PR were higher in immunohistochemistry. Ki-67 index has been suggested as a potential indicator of malignant potential and poor outcome of SPNs [23-25], but some study regarded the fact that it was not associated with malignancy [15]. The low Ki-67 index $(\leq 5 \%)$ indicates a slow growth of the tumors. Ki-67 immunoreactivity proliferative index less than $1 \%$ of 4 malignant cases in our study had a good survival. The low proliferative index of Ki-67 may predict favorable outcome of malignant SPN. Regretfully, two patients died of SPN that were not detected by Ki- 67 .

We recommend that surgeons consider a more radical resection with an incomplete capsule of tumor. Papavramidis and Papavramidis [1] summarized the survival data in 467 patients and reported the 2-year and 5-year survival rate were 97\% and 95\%. Law et al. summarized the 1952 cases with recurrence and death of SPN, and $4.4 \%$ patients suffered recurrence; meanwhile $1.5 \%$ patients died of SPN. Similar to their findings, the 5-year disease-specific survival rate was $95.7 \%$ and recurrence rate was $1.9 \%$ in our study. Martin et al. [26] found that microscopic positive margin, invasion of surrounding structures, and size $>5 \mathrm{~cm}$ were not significant predictors of survival.

In summary, SPN is a rare pancreatic tumor, with a lowgrade malignancy and strong female predilection. Clinical manifestations have no specificity, imaging examination is contributed by tumor location, and diagnosis relies on pathology. Surgery is the main method of treatment and the prognosis is good. Incomplete capsule may suggest a 
malignant SPN and a prognostic indicator of disease-specific survival.

\section{Conflicts of Interest}

The authors declare that they have no conflicts of interest.

\section{Acknowledgments}

This work was supported by Chinese National Science Foundation (no. 81672835) to Ming Dong.

\section{References}

[1] T. Papavramidis and S. Papavramidis, "Solid pseudopapillary tumors of the pancreas: review of 718 patients reported in english literature," Journal of the American College of Surgeons, vol. 200, no. 6, pp. 965-972, 2005.

[2] V. Frantz, Tumor of the pancreas, 1959, p. 32-33.

[3] J. K. Law, A. Ahmed, V. K. Singh et al., "A systematic review of solid-pseudopapillary neoplasms: are these rare lesions?" Pancreas, vol. 43, no. 3, pp. 331-337, 2014.

[4] B. K. P. Goh, Y.-M. Tan, P.-C. Cheow et al., "Solid pseudopapillary neoplasms of the pancreas: an updated experience," Journal of Surgical Oncology, vol. 95, no. 8, pp. 640-644, 2007.

[5] T. Morikawa, T. Onogawa, S. Maeda et al., "Solid pseudopapillary neoplasms of the pancreas: an 18-year experience at a single Japanese Institution," Surgery Today, vol. 43, no. 1, pp. 26-32, 2013.

[6] J. Hwang, D. Y. Kim, S. C. Kim, J.-M. Namgoong, and S.M. Hong, "Solid-pseudopapillary neoplasm of the pancreas in children: can we predict malignancy?" Journal of Pediatric Surgery, vol. 49, no. 12, pp. 1730-1733, 2014.

[7] C. M. Kang, S. H. Choi, S. C. Kim, W. J. Lee, D. W. Choi, and S. W. Kim, "Predicting recurrence of pancreatic solid pseudopapillary tumors after surgical resection: a multicenter analysis in Korea," Annals of Surgery, vol. 260, no. 2, pp. 348355, 2014.

[8] Y.-W. Tien, K.-H. Ser, R.-H. Hu, C.-Y. Lee, Y.-M. Jeng, and P.H. Lee, "Solid pseudopapillary neoplasms of the pancreas: is there a pathologic basis for the observed gender differences in incidence?” Surgery, vol. 137, no. 6, pp. 591-596, 2005.

[9] S. G. Uppin, M. Hui, V. Thumma et al., "Solid-pseudopapillary neoplasm of the pancreas: a clinicopathological and immunohistochemical study of 33 cases from a single institution in Southern India," Indian Journal of Pathology and Microbiology, vol. 58, no. 2, pp. 163-169, 2015.

[10] M. C. C. Machado, M. A. C. Machado, T. Bacchella, J. Jukemura, J. L. Almeida, and J. E. M. Cunha, "Solid pseudopapillary neoplasm of the pancreas: distinct patterns of onset, diagnosis, and prognosis for male versus female patients," Surgery, vol. 143, no. 1, pp. 29-34, 2008.

[11] G. Sun, C. Chen, J. Yao, H. Shi, Y. He, and W. Z, "Diagnosis and treatment of solid pseudopapillary tumor of pancreas: a report of 8 cases with review of domestic literature," Chinese Journal of General Surgery, pp. 902-907, 2008.

[12] M. H. Yu, J. Y. Lee, M. A. Kim et al., "MR imaging features of small solid pseudopapillary tumors: retrospective differentiation from other small solid pancreatic tumors," American Journal of Roentgenology, vol. 195, no. 6, pp. 1324-1332, 2010.
[13] Q. Yin, M. Wang, C. Wang et al., "Differentiation between benign and malignant solid pseudopapillary tumor of the pancreas by MDCT,' European Journal of Radiology, vol. 81, no. 11, pp. 3010-3018, 2012.

[14] M. A. Khashab, K. Kim, A. M. Lennon et al., "Should we do EUS/FNA on patients with pancreatic cysts? the incremental diagnostic yield of EUS over CT/MRI for prediction of cystic neoplasms," Pancreas, vol. 42, no. 4, pp. 717-721, 2013.

[15] I. Hosokawa, H. Shimizu, M. Ohtsuka et al., "Preoperative diagnosis and surgical management for solid pseudopapillary neoplasm of the pancreas," Journal of Hepato-Biliary-Pancreatic Sciences, vol. 21, no. 8, pp. 573-578, 2014.

[16] P. O. Fais, E. Carricaburu, S. Sarnacki et al., "Is laparoscopic management suitable for solid pseudo-papillary tumors of the pancreas?" Pediatric Surgery International, vol. 25, no. 7, pp. 617621, 2009.

[17] Y. Guerrache, P. Soyer, A. Dohan et al., "Solid-pseudopapillary tumor of the pancreas: MR imaging findings in 21 patients," Clinical Imaging, vol. 38, no. 4, pp. 475-482, 2014.

[18] Y. E. Chung, M.-J. Kim, J.-Y. Choi et al., "Differentiation of benign and malignant solid pseudopapillary neoplasms of the pancreas," Journal of Computer Assisted Tomography, vol. 33, no. 5, pp. 689-694, 2009.

[19] W. Cheuk, I. Beavon, D. T. Y. Chui, and J. K. C. Chan, "Extrapancreatic solid pseudopapillary neoplasm: report of a case of primary ovarian origin and review of the literature," International Journal of Gynecological Pathology, vol. 30, no. 6, pp. 539-543, 2011.

[20] M. Michal, S. Bulimbasic, M. Coric et al., "Pancreatic analogue solid pseudopapillary neoplasm arising in the paratesticular location. The first case report," Human Pathology, vol. 56, pp. 52-56, 2016.

[21] L. Naar, D.-A. Spanomichou, A. Mastoraki, V. Smyrniotis, and N. Arkadopoulos, "Solid pseudopapillary neoplasms of the pancreas: a surgical and genetic enigma," World Journal of Surgery, vol. 41, no. 7, pp. 1871-1881, 2017.

[22] C. Geers, M. Pierre, G. Jean-François et al., "Solid and pseudopapillary tumor of the pancreas-review and new insights into pathogenesis," American Journal of Surgical Pathology, vol. 30, no. 10, pp. 1243-1249, 2006.

[23] S. Serra and R. Chetty, "Revision 2: an immunohistochemical approach and evaluation of solid pseudopapillary tumour of the pancreas," Journal of Clinical Pathology, vol. 61, no. 11, pp. 1153$1159,2008$.

[24] F. Yang, C. Jin, J. Long et al., "Solid pseudopapillary tumor of the pancreas: a case series of 26 consecutive patients," The American Journal of Surgery, vol. 198, no. 2, pp. 210-215, 2009.

[25] Y. Watanabe, K. Okamoto, K. Okada, M. Aikawa, I. Koyama, and H. Yamaguchi, "A case of aggressive solid pseudopapillary neoplasm: comparison of clinical and pathologic features with non-aggressive cases," Pathology International, vol. 67, no. 4, pp. 202-207, 2017.

[26] R. C. G. Martin, D. S. Klimstra, M. F. Brennan, and K. C. Conlon, "Solid-pseudopapillary tumor of the pancreas: a surgical enigma?" Annals of Surgical Oncology, vol. 9, no. 1, pp. $35-40,2002$. 


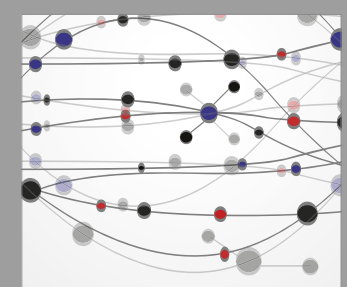

The Scientific World Journal
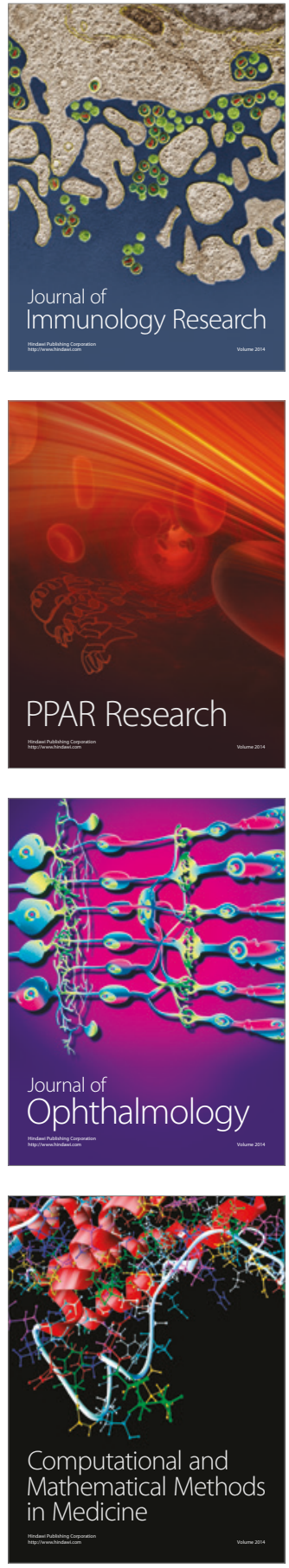

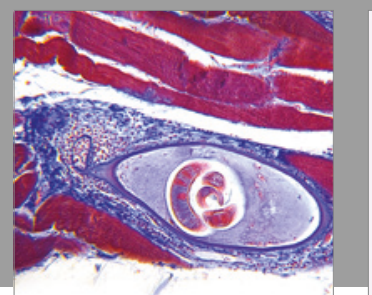

Gastroenterology Research and Practice
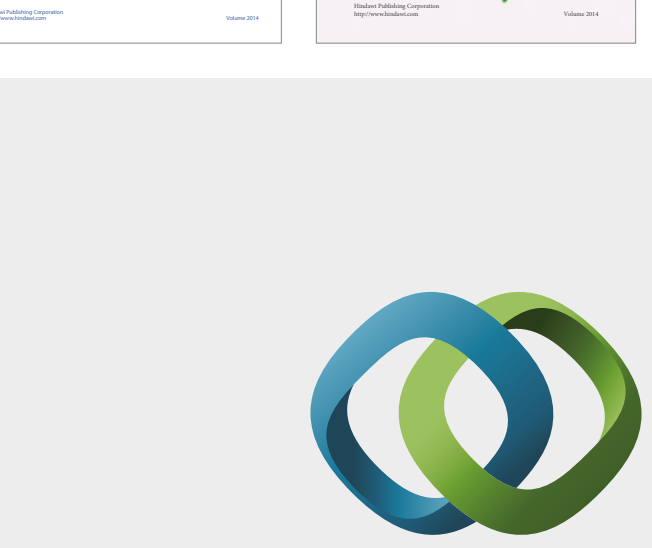

\section{Hindawi}

Submit your manuscripts at

https://www.hindawi.com
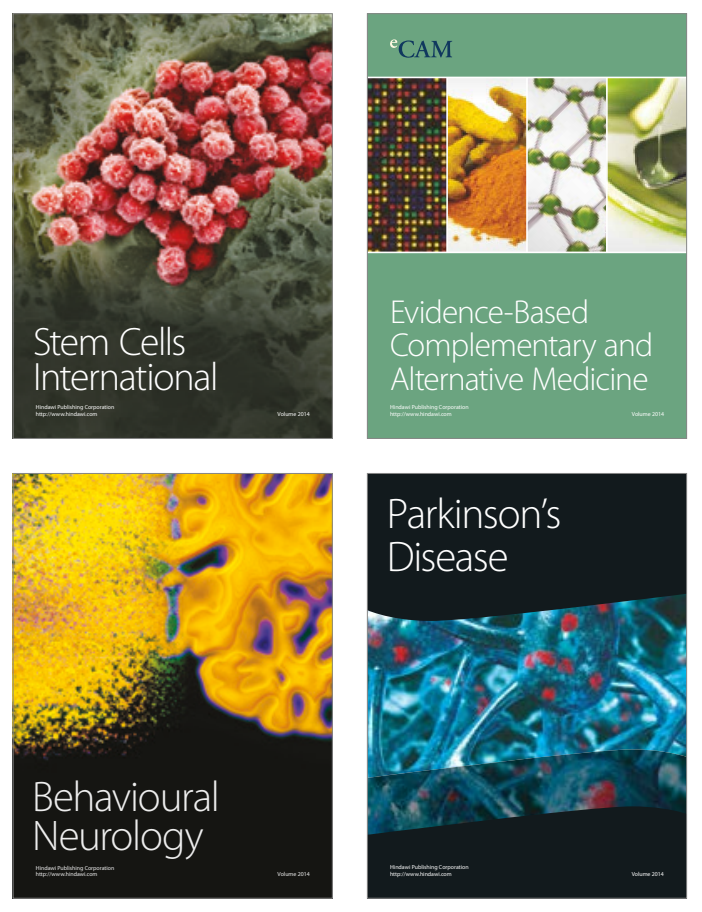
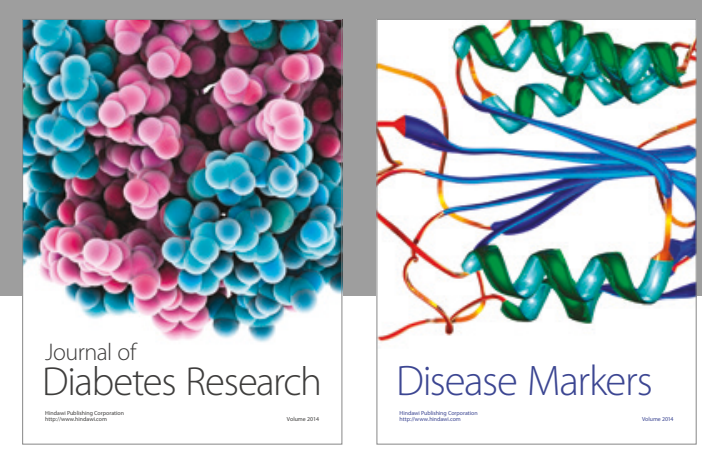

Disease Markers
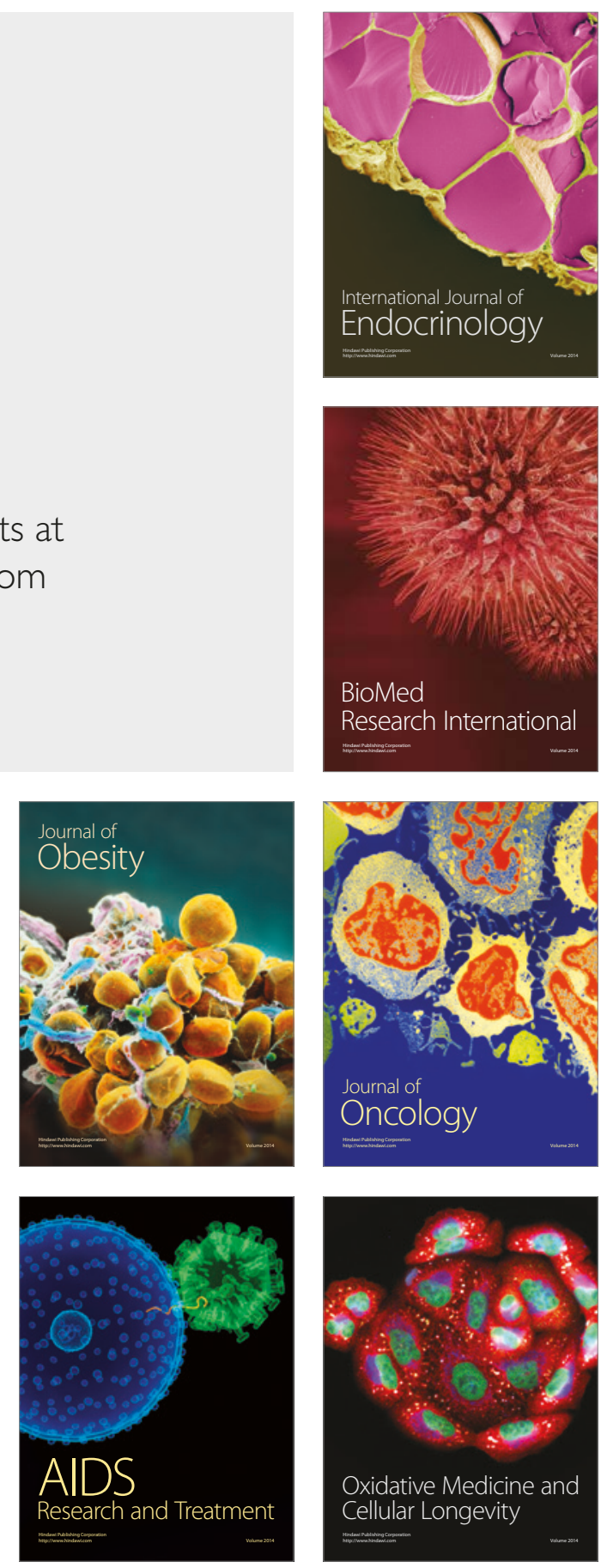